\title{
Post-traumatic Stress Disorder Is Associated With Irritable Bowel Syndrome in African Americans
}

\author{
Natalya Iorio, ${ }^{*}$ Kian Makipour, Amiya Palit and Frank K Friedenberg \\ Digestive Disease Center, Temple University School of Medicine, Philadelphia, PA, USA
}

\begin{abstract}
Background/Aims
Psychosocial stressors likely play an important role in irritable bowel syndrome (IBS). The association between IBS and post-traumatic stress disorder (PTSD) in non-minorities has been described. Our aim was to investigate the potential association between IBS and PTSD in an urban African American population.
\end{abstract}

\section{Methods}

Our institution maintains a longitudinal population-based survey of African Americans (AA). The survey utilizes a complex, stratified sampling design. The study group consisted of adult AA meeting Rome III criteria for IBS of any subtype. The 4-item Primary Care PTSD screener was administered; score of $\geq 3$ (range, 0-4) was considered positive for PTSD. Depression (Public Health Questionnaire-9 depression) and anxiety (generalized anxiety disorder-7) levels were measured using standardized scales. To assess quality of life, norm-based physical and mental component summary scores from the short-form 36 health survey version 2 were obtained. Descriptive and inferential statistics were calculated using Complex Sample Module of SPSS after weighting of the study sample.

\section{Results}

Four hundred nineteen subjects included corresponded to a weighted $21,264(95 \% \mathrm{Cl}, 19,777-22,751)$ individuals. The prevalence of IBS in our sample of urban AA was $8.2 \%$. In multivariate regression analysis, female gender, age $>40$, higher educational attainment and divorce were independently associated with IBS. Those with IBS were considerably more likely to suffer from PTSD (OR, 4.54; 95\% Cl, 4.07-5.06). PTSD was independently associated with depression, anxiety, harmful drinking and substance abuse.

\section{Conclusions}

In AA, PTSD is independently associated with IBS. PTSD has a significantly negative impact on physical and mental self-assessment of quality of life. Evaluation of minorities presenting with functional gastrointestinal disorders should include screening for PTSD.

\section{(J Neurogastroenterol Motil 2014;20:523-530)}

Key Words

African Americans; Irritable bowel syndrome; Rome; Stress disorders, post-traumatic

Received: April 3, 2014 Revised: June 5, 2014 Accepted: June 11, 2014

(c) This is an Open Access article distributed under the terms of the Creative Commons Attribution Non-Commercial License (http://creativecommons. org/licenses/by-nc/3.0) which permits unrestricted non-commercial use, distribution, and reproduction in any medium, provided the original work is properly cited.

${ }^{*}$ Correspondence: Natalya lorio, MD

Temple University Hospital, 3401 North Broad Street, Philadelphia, PA 19140, USA

Tel: +1-215-707-4391, Fax: +1-215-707-2684, E-mail: Frank.Friedenberg@tuhs.temple.edu

Financial support: This study was supported by DHHS (Grant No. K24DK083268).

Conflicts of interest: None.

Author contributions: Natalya lorio and Kian Makipour, drafted manuscript and literature review; Amiya Palit, data collection and database maintenance; Frank K Friedenberg, study conception, statistical analysis and drafted manuscript. 


\section{Introduction}

Irritable bowel syndrome (IBS) is a functional gastrointestinal disorder characterized by abdominal discomfort and change in bowel habit that accounts for symptoms affecting 30-45 million US adults each year. ${ }^{1}$ The prevalence of IBS in North America has been estimated at $3-20 \%$ with a majority of studies reporting $10-15 \%{ }^{2,3} \mathrm{~A}$ similar prevalence of $11.5 \%$ is reported in Western Europe. ${ }^{4}$ Two thirds of IBS sufferers are women. ${ }^{2,5}$ The peak age for IBS symptoms is between 35-44 years. ${ }^{2}$ Women develop symptoms at an earlier age than men (25.6 vs. 30.5 years, $P=0.069$ ). ${ }^{6}$ Caucasians are reportedly 2.5 times more likely (95\% confidence interval (CI), 1.5-4.0) than African Americans (AA) to have IBS. ${ }^{7}$ However, overall prevalence of IBS by ethnic groups in the US has not been reported. ${ }^{8}$

The impact of IBS varies over time with respect to severity and symptom pattern, both of which affect quality of life (QOL). ${ }^{9}$ The most commonly reported symptoms include abdominal pain $(88 \%)$, bloating $(80 \%)$, tiredness $(60 \%)$, diarrhea $(59 \%)$ and constipation (53\%). ${ }^{4}$ IBS sufferers miss approximately twice as many work days per year due to symptoms compared with non-sufferers. ${ }^{2,4}$ People with IBS also more frequently report fatigue $(21.1 \%$ vs. $9.9 \%, P=0.001)$ and lack of energy (79\% vs. $59.5 \%, P=0.000) .{ }^{10}$ IBS has been shown to have a negative impact on QOL in several domains including physical functioning, bodily pain, general health, vitality, social functioning and mental health. ${ }^{5,11,12}$

Psychosocial stressors play an important role in IBS from predisposition to the disorder to precipitation of symptoms. ${ }^{3,12,13}$ IBS is associated with being unemployed, unmarried and having a lower income. ${ }^{5}$ The use of psychotropic medications for the treatment of IBS is common, particularly tricyclic antidepressants, selective serotonin reuptake inhibitors and serotonin-norepinephrine reuptake inhibitors. ${ }^{3,14,15}$ An important condition potentially associated with IBS is post-traumatic stress disorder (PTSD). PTSD is defined as an exposure to a traumatic physical or emotional event that causes re-experience of the occurrence, avoidance of triggers, development of negative thoughts and moods, and chronic hyper-arousal symptoms. ${ }^{16}$ Often PTSD is the result of childhood sexual abuse. ${ }^{17,18}$ Patients with functional gastrointestinal disorders are more likely to have experienced severe abuse or life threat than those with structural disease. ${ }^{14}$

In our review of the literature we were unable to find data on the association between PTSD and IBS in the AA population.
Our aim was to investigate the risk factors for IBS and PTSD among AAs and the impact on QOL. Our institution maintains a longitudinal population-based survey of urban AA which allows for investigation of this association while controlling for many relevant confounders.

\section{Materials and Methods}

Temple Registry for the Investigation of African American Gastrointestinal Disease Epidemiology (TRIAGE) is an ongoing cohort study of AA residing within the ZIP Code Tabulation Area (ZCTA) incorporating Temple University Hospital. Survey design has been described in detail previously. ${ }^{19}$ In summary, our population-based survey utilizes a complex, stratified sampling design. In order to obtain a representative sample of the entire AA community we carefully identified 4 targeted survey zones. We hand-delivered invitations to 1,000 individual dwellings. The invitation stated that to participate in the survey the subject had to be self-described as an AA, older than 18 and a resident of the dwelling for at least 3 years. Only one adult per household could participate. All surveys are conducted at our research department. A research coordinator records demographic information and measures the subject's height, weight, hip and waist circumference. Patients are then seated at a computer terminal. Our survey was developed using Microsoft Access 2007 (Microsoft Corporation, Redmond, WA, USA). A coordinator assists participants with visual impairment and those unable to read at the fifth grade level. Subjects progress through the survey by selecting choices using a computer mouse. Subjects cannot progress through the survey without answering all questions. The survey was approved by our Institutional Review Board. The trial is registered on clinicaltrials.gov (NCT01262755). The results reported here are from the entry survey subjects completed.

\section{Survey Components}

\section{Dependent variable}

The outcome variable of interest was the presence of IBS as defined by Rome III criteria. ${ }^{20}$ For analysis purposes, individuals meeting IBS criteria were considered in aggregate regardless of subtype. Subjects were not required to have had a colonoscopy or other testing to be included.

\section{Independent variables}

The survey queries the subject's marital status, highest edu- 
cational attainment, annual income, medical history and a list of up to 10 prescriptions and over-the-counter medications. To assess for addictive behaviors, subjects completed the Alcohol Use Disorders Identification Test (AUDIT), Drug Abuse Screening Test (DAST) and the Fagerstrom Test for Nicotine Dependence (FTND). Depression (Public Health Questionnaire-9 depression) and anxiety levels (generalized anxiety disorder-7) were assessed using validated instruments. The primary care PTSD (PC-PTSD) screener was administered and a score of $\geq 3$ (range, 0-4) was considered "highly likely positive" for PTSD. ${ }^{21,22}$ All subjects completed the medical outcomes study 36-item short-form health survey (SF-36) version 2, a patient-reported health survey consisting of questions grouped into 8 domains: vitality, physical functioning, bodily pain, general health perceptions, physical role functioning, emotional role functioning, social role functioning and mental health. ${ }^{23}$ Each domain is scored from 0 -100 with the higher score (100) corresponding to less impairment. $^{23}$

\section{Survey Analysis}

\section{Sample weighting}

Two levels of weighting were performed. An initial weight is applied to adjust for the unequal probability of selection within the household. For second-level weighting, we utilize data from the 2010 US Census which stratifies AA living in the ZCTA by sex and age. An additional scaling variable was used to estimate population-level prevalence data. The scale variable (v) represented the ratio of the entire AA population of the ZCTA divided by the number of subjects interviewed $\left(\mathrm{v}=\mathrm{N}_{\mathrm{B}} / \mathrm{n}_{\mathrm{B}}\right)$.

\section{Sample size}

We estimated that the prevalence of IBS would be $7-10 \%$ in the community. ${ }^{24} \mathrm{We}$ anticipated that our final regression model would have 3-4 independent predictors. To develop a well-calibrated regression model with $\geq 8$ outcome events per predictor variable, our protocol called for the recruitment of a minimum of 400 subjects.

\section{Statistical Methods}

We designed a complex sample file plan using the Complex Sample Module of SPSS 19.0 (IBM SPSS, Armonk, New York, USA). Descriptive and inferential statistics were determined using the Complex Sample Module yielding point estimates with 95\% CI. For categorical variables, an adjusted F statistic was calculated. General linear models were used to estimate the association of continuous predictor variables with categorical outcomes. $P$-values were calculated based on the Wald statistic. A multivariable logistic regression was performed to identify variables independently associated with the prevalence of IBS. Odds ratios (OR) along with their corresponding 95\% CI were calculated for variables included in the model. All hypothesis testing was 2-tailed with $\alpha=0.05$.

\section{Results}

We recruited 419 subjects from unique households in the targeted zip code tabulation area. After weighting and scaling of the surveyed sample this corresponded to 21,264 (95\% CI, 19,777-22,751) citizens. The overall weighted prevalence of IBS was $8.2 \%$. Table 1 highlights the characteristics of the population stratified by IBS status. The number of females in the group with IBS was substantially higher than in the group without $81.8 \%$ (95\% CI, 66.4-99.1) vs. 54.5\% (95\% CI, 48.3-60.6). IBS subjects were more obese and more likely to have undergone divorce. Those with IBS had a higher level of educational attainment but minimal difference in annual income. Not surprisingly, those with IBS were more likely to have been investigated with colonoscopy $(35.1 \%$ [95\% CI, 17.7-57.5] vs. $23.1 \%$ [95\% CI, 17.7-29.7]), however the percent of individuals visiting a doctor at least once in the previous year was similar. By univariate analysis the prevalence of PTSD (score $\geq 3$ ) and harmful alcohol use was far higher in those with IBS than those without.

Table 2 demonstrates that the majority of individuals with IBS suffered from moderate-severe depression (73\%), and $17.3 \%$ had severe depression (95\% CI, 7.3-35.5). More patients with IBS than without reported taking a prescription or herbal antidepressant ( $13.5 \%$ vs. $8.3 \%$ ). Although less common than depression, $51 \%$ of individuals with IBS had some anxiety which is substantially higher than individuals without IBS (34.1\%). Figure demonstrates that IBS has a negative impact on every domain of QOL as measured by the SF-36. Table 3 shows the results of a multivariable regression model. Female gender, age $>40$, higher educational attainment and divorce were independently associated with IBS. Those with IBS were 4.5 times more likely to suffer from PTSD (OR, 4.54; 95\% CI, 4.07-5.06).

We further explored the impact of PTSD on our study population. As shown in Table 4, the majority of subjects who met criteria for PTSD were female (70.4\%). The negative impact of PTSD was associated with depression, anxiety, harmful drinking 
Table 1. Characteristics of Study Subjects Stratified by Irritable Bowel Syndrome Status

\begin{tabular}{|c|c|c|c|}
\hline & $\begin{array}{c}\text { Total } \\
\text { Weighted }(\mathrm{N}=21,264) \\
(95 \% \mathrm{CI}: 19,777-22,751)\end{array}$ & $\begin{array}{c}\text { No IBS } \\
\text { Weighted }(\mathrm{n}=19,516) \\
(95 \% \text { CI: } 17,974-21,058)\end{array}$ & $\begin{array}{c}\text { IBS } \\
\text { Weighted }(\mathrm{n}=1,748) \\
(95 \% \text { CI: } 1,065-2,431)\end{array}$ \\
\hline Age (yr) & $44.2(42.0-46.4)$ & $44.0(41.7-46.3)$ & $46.3(39.9-52.8)$ \\
\hline$\%$ Female & $56.8(50.8-62.5)$ & $54.5(48.3-60.6)$ & $81.8(66.4-99.1)$ \\
\hline Body mass index & $29.1(28.2-30.0)$ & $28.2(28.0-29.8)$ & $30.9(26.5-35.2)$ \\
\hline \multicolumn{4}{|l|}{ Educational level (\%) } \\
\hline$<$ High school graduate & $31.6(26.4-37.3)$ & $33.0(27.4-39.0)$ & $16.5(8.0-31.0)$ \\
\hline High school graduate & $61.8(55.9-67.4)$ & $60.9(54.6-66.7)$ & $72.7(53.2-86.2)$ \\
\hline College graduate & $6.6(4.1-10.4)$ & $6.2(3.7-10.1)$ & $10.8(2.7-34.7)$ \\
\hline \multicolumn{4}{|l|}{ Marital status (\%) } \\
\hline Single, never married & $73.4(67.2-78.9)$ & $73.8(67.1-79.5)$ & $69.7(51.7-83.2)$ \\
\hline Divorced & $14.1(9.7-20.1)$ & $13.6(9.0-20.2)$ & $19.3(9.0-36.7)$ \\
\hline Separated & $4.0(2.2-7.3)$ & $4.1(2.1-7.8)$ & $2.9(0.9-9.1)$ \\
\hline Married & $8.4(5.7-12.4)$ & $8.5(5.5-12.7)$ & $8.0(2.9-20.4)$ \\
\hline \multicolumn{4}{|l|}{ Annual home employment income (\%) } \\
\hline Unemployed & $35.5(30.3-41.1)$ & $35.5(30.0-41.4)$ & $35.9(20.7-54.5)$ \\
\hline$<\$ 20,000$ & $48.2(42.3-54.2)$ & $48.1(41.9-54.4)$ & $49.3(30.2-68.6)$ \\
\hline$\$ 20,000-\$ 50,000$ & $14.7(10.9-19.6)$ & $14.9(10.9-20.1)$ & $12.6(4.6-30.2)$ \\
\hline$>\$ 50,000$ & $1.5(0.9-2.8)$ & $1.5(0.8-2.7)$ & $2.2(0.3-14.6)$ \\
\hline Visit PCP at least yearly (\%) & $79.6(74.5-83.8)$ & $79.3(74.0-83.8)$ & $81.9(65.0-91.7)$ \\
\hline Colonoscopy in past 5 years (\%) & $24.1(18.8-30.4)$ & $23.1(17.7-29.7)$ & $35.1(17.7-57.5)$ \\
\hline \multicolumn{4}{|l|}{ Comorbidities } \\
\hline Hypertension & $38.9(33.1-45.1)$ & $39.0(32.9-45.4)$ & $38.6(20.5-60.5)$ \\
\hline Obstructive airways disease & $26.3(21.2-32.1)$ & $25.4(20.1-31.6)$ & $35.9(19.9-55.8)$ \\
\hline Diabetes & $15.0(10.9-20.2)$ & $15.1(10.8-20.6)$ & $13.8(5.3-31.3)$ \\
\hline Elevated cholesterol & $20.4(15.8-25.8)$ & $20.8(15.9-26.6)$ & $15.7(6.9-31.8)$ \\
\hline Coronary artery disease & $4.0(2.0-7.9)$ & $4.0(1.9-8.3)$ & $4.0(1.0-15.2)$ \\
\hline Viral hepatitis & $4.1(2.2-7.5)$ & $4.2(2.2-7.9)$ & $2.9(0.4-18.1)$ \\
\hline \multicolumn{4}{|l|}{ PTSD screener score (\%) } \\
\hline 0 & $59.1(53.2-64.7)$ & $61.2(55.1-67.0)$ & $35.2(17.7-57.7)$ \\
\hline 1 & $9.0(6.0-13.2)$ & $9.2(6.1-13.6)$ & $6.5(0.9-34)$ \\
\hline 2 & $9.7(6.7-13.9)$ & $10.1(6.8-14.5)$ & $5.7(1.4-21.1)$ \\
\hline 3 & $8.0(5.6-11.4)$ & $7.7(5.2-11.2)$ & $12.6(4.7-29.7)$ \\
\hline 4 & $14.2(10.7-18.5)$ & $11.8(8.5-16.2)$ & $40.0(23.6-59.1)$ \\
\hline Harmful drinking habits (\%) & $12.1(9.1-15.8)$ & $11.4(8.4-15.3)$ & $19.9(9.2-37.7)$ \\
\hline High nicotine dependence (\%) & $5.1(3.1-8.3)$ & $5.4(3.3-8.8)$ & $2.0(0.3-13.5)$ \\
\hline Prescription or illicit substance abuse (\%) & $11.1(8.1-14.9)$ & $11.2(8.1-15.4)$ & $9.0(3.4-21.7)$ \\
\hline
\end{tabular}

$\mathrm{PCP}$, primary care physician.

and substance abuse. The component QOL summary scores from the SF-36 were significantly reduced for the mental (39.2 \pm 11.1 vs. $54.6 \pm 9.9)$ and physical $(42.9 \pm 10.2$ vs. $47.6 \pm$ 10.2) domains in those with PTSD.

\section{Discussion}

In this study we found the prevalence of IBS among urban AA to be $8.2 \%$ with the majority $(81.8 \%)$ female. PTSD had a strong independent association with IBS among AA (OR, 4.54; 95\% CI, 4.07-5.06). Similarly, we found a strong association between PTSD and moderately severe to severe depression as well as anti-depressant use. In distinction from previous reports, we found age $\geq 40$ years to be associated with greater than twice the risk of IBS. Previous studies reported a higher prevalence of IBS in those less than 45 years old ${ }^{2,5}$ and only $9.4 \%$ in those older than 45 years. ${ }^{1}$ The prevalence of women with IBS is higher in our study $81.8 \%$ (95\% CI, 66.4-99.1) compared to the commonly re- 
Table 2. Depression and Anxiety Prevalence Stratified by Irritable Bowel Syndrome Status

\begin{tabular}{lccc}
\hline & $\begin{array}{c}\text { Total weighted }(\mathrm{N}=21,264) \\
(95 \% \mathrm{CI}, 19,777-22,751)\end{array}$ & $\begin{array}{c}\text { No IBS weighted }(\mathrm{n}=19,516) \\
(95 \% \text { CI, 17,974-21,058) }\end{array}$ & $\begin{array}{c}\text { IBS weighted }(\mathrm{n}=1,748) \\
(95 \% \mathrm{CI}, 1,065-2,431)\end{array}$ \\
\hline $\begin{array}{l}\text { Depression level } \\
\text { None-mild }\end{array}$ & $62.3(56.5-67.8)$ & $65.3(59.3-70.9)$ & $26.8(10.4-53.7)$ \\
Moderate & $18.9(14.7-24.0)$ & $19.1(14.7-24.5)$ & $17.0(8.1-42.2)$ \\
Moderately severe & $12.5(9.3-16.7)$ & $10.3(7.3-14.3)$ & $38.9(21.7-59.5)$ \\
Severe & $6.2(4.2-9.3)$ & $5.3(3.3-8.4)$ & $17.3(7.3-35.5)$ \\
Prescribed an antidepressant & $8.7(6.3-12.0)$ & $8.3(5.8-11.8)$ & $13.5(6.0-27.8)$ \\
Anxiety disability & & & $49.2(30.1-68.5)$ \\
None & $64.5(58.8-69.9)$ & $65.9(59.9-71.4)$ & $41.7(24.6-60.9)$ \\
Some amount & $28.1(23.2-33.6)$ & $26.9(21.8-32.7)$ & $9.1(3.5-21.8)$ \\
Moderate amount & $5.5(3.5-8.4)$ & $5.1(3.2-8.3)$ & $0.0(0.0-41.9)$ \\
Severe amount & $1.9(0.9-4.1)$ & $2.1(0.9-4.4)$ & \\
\hline
\end{tabular}

IBS, irritable bowel syndrome.

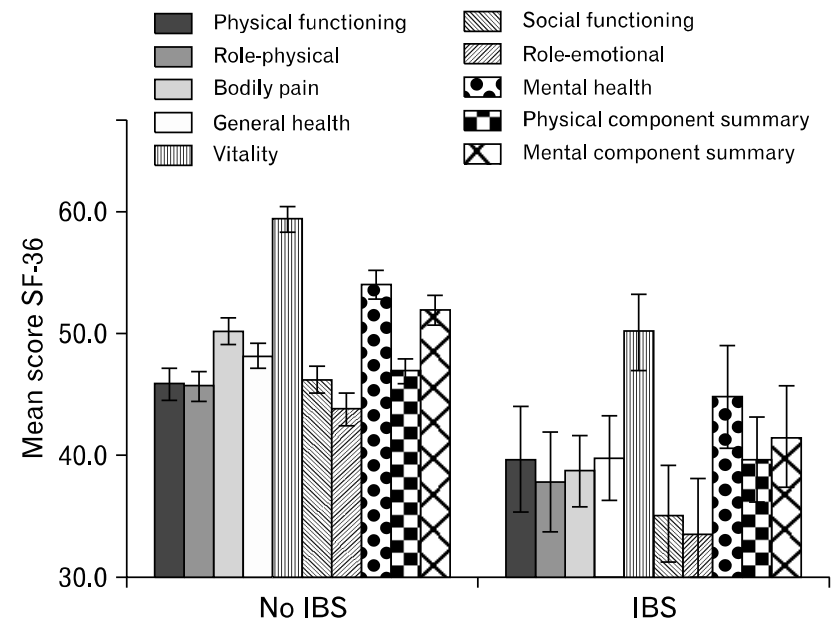

Figure. Comparison of 36-item short-form health survey (SF-36) scores in patients with and without irritable bowel syndrome (IBS).

ported $2: 1$ ratio. $^{2,5}$

To our knowledge, no prior studies have evaluated PTSD and its association with IBS among AA. In a survey of 990 subjects (670 AA and 320 Caucasian) Minocha et $\mathrm{al}^{10}$ found no difference in history of abuse (physical or sexual) among AA subjects with IBS. They found an overall increased frequency of abuse history among subjects with IBS as well as more suicidal thoughts, loss of interest, anxiety or depression. ${ }^{10}$ Drossman ${ }^{14}$ hypothesized that higher abuse rates correlated with more severe symptoms.

Alcohol abuse is strongly linked to various emotional, psychological and physical illnesses. We found an increased prevalence of harmful alcohol abuse among subjects with IBS and a
Table 3. Regression Model - Independent Factors Associated With Irritable Bowel Syndrome

\begin{tabular}{lcc}
\hline & Adjusted $^{\mathrm{a}} \mathrm{OR}$ & $95 \%$ CI for OR \\
\hline Male & Reference & \\
Female & 3.28 & $2.88-3.74$ \\
Age $<40$ & Reference & \\
Age $\geq 40$ & 2.21 & $1.95-2.50$ \\
Education & & \\
$\quad<$ High school graduate & Reference & \\
$\quad$ High school graduate & 3.25 & $2.82-3.73$ \\
$\quad$ College graduate & 4.97 & $3.99-6.19$ \\
Marital status & & \\
$\quad$ Single, never married & Reference & \\
$\quad$ Divorced & 1.38 & $1.19-1.60$ \\
$\quad$ Married & 0.67 & $0.54-0.82$ \\
Annual home employment income & & \\
$\quad$ Unemployed & Reference & \\
$\quad<\quad \$ 20,000$ & 0.86 & $0.76-0.97$ \\
$\quad>\quad \$ 0,000$ & 1.26 & $0.87-1.82$ \\
Screen for PTSD & & \\
$\quad$ Negative & Reference & \\
Positive & 4.54 & $4.07-5.06$ \\
\hline
\end{tabular}

${ }^{a}$ Adjusted for all other variables in model.

PTSD, post-traumatic stress disorder.

more than 2-fold risk among subjects with PTSD (20.0\% vs. 9.8\%; OR, 2.29; 95\% CI, 2.10-2.50). Similarly, there was a higher rate of substance abuse among subjects with PTSD ( $22.3 \%$ vs. $8.0 \%)$, but only a slightly higher rate comparing IBS vs. non-IBS (11.2\% vs. 9\%). In a prospective community-based study of women (88\% non-Hispanic White) aged $18-48$ years 
Table 4. Univariate Relationship Between Select Variables and Post-traumatic Stress Disorder Status

\begin{tabular}{|c|c|c|c|c|}
\hline & \multicolumn{2}{|c|}{ PTSD Status } & \multirow[b]{2}{*}{$\mathrm{OR}^{\mathrm{a}}(95 \% \mathrm{CI})$} & \multirow[b]{2}{*}{$P$-value } \\
\hline & $\begin{array}{l}\text { Positive Weighted } \\
\quad(n=4,729)\end{array}$ & $\begin{array}{l}\text { Negative Weighted } \\
\quad(\mathrm{n}=16,535)\end{array}$ & & \\
\hline \multicolumn{5}{|l|}{ Categorical variables (\%) } \\
\hline Female & 70.4 & 52.9 & $2.12(1.98-2.27)$ & \\
\hline Divorce rate & 11.9 & 14.7 & $0.78(0.71-0.86)$ & \\
\hline Anxiety disability & 18.0 & 4.3 & $4.86(4.38-5.30)$ & \\
\hline Depression & 55.2 & 8.9 & $12.61(11.65-13.66)$ & \\
\hline Harmful alcohol use & 20.0 & 9.8 & $2.29(2.10-2.50)$ & \\
\hline Substance abuse & 22.3 & 8.0 & $3.32(3.02-3.64)$ & \\
\hline Unemployment & 37.8 & 34.9 & $1.14(1.06-1.21)$ & \\
\hline \multicolumn{5}{|c|}{ Continuous variables mean (SD) } \\
\hline PCS & $42.9(10.2)$ & $47.6(10.2)$ & & $<0.001$ \\
\hline MCS & $39.2(11.1)$ & $54.6(9.9)$ & & $<0.001$ \\
\hline
\end{tabular}

${ }^{a}$ Using the Cochran's Mantel-Haenszel test of conditional independence.

PTSD, post-traumatic stress disorder; Depression, individuals self-assessed as moderately to severely depressed; Anxiety disability, individuals self-assessed as moderate to severe; PCS, physical component summary; MCS, mental component summary.

with and without IBS, Reding et $\mathrm{al}^{24}$ studied the relationship between alcohol consumption and GI symptoms and found no difference in the quantity of alcohol consumption between women with and without IBS. However, those that reported binge drinking were twice as likely to experience diarrhea (OR, 2.1; 95\% CI, 1.2-3.5), nausea (OR, 2.4; 95\% CI, 1.2-4.8), abdominal pain $(\mathrm{OR}, 2.1 ; 95 \% \mathrm{CI}, 1.2-3.7)$ and indigestion $(\mathrm{OR}, 2.0 ; 95 \% \mathrm{CI}$, 1.1-3.6) the following day. However, Halder et $\mathrm{al}^{25}$ found no significant association between IBS and alcohol consumption when accounting for other variables such as gender, smoking, education, age, and somatization.

There are several avenues by which IBS could be linked to PTSD. The pathogenesis of IBS is multifactorial with psychosocial, genetic, nervous system (central and enteric), hormonal, visceral hypersensitivity and infectious/inflammatory components. ${ }^{3,11,15}$ This concept is presented in the "biopsychosocial" model of IBS by Tanaka et al. ${ }^{12}$ They divide the factors that influence IBS development into early life (genetics, social learning), psychosocial (stress, abuse and psychological state) and abnormal physiology (including abnormalities of motility and sensitivity, brain-gut interaction, regional brain activation, hypothalamic-pituitary-adrenal axis [HPAA] and post-infectious). Videlock et $\mathrm{al}^{26}$ studied the HPAA response to a visceral stressor (sigmoidoscopy) in patients with IBS vs. healthy controls and those exposed to early adverse life events (EALs) using salivary cortisol levels. They found higher mean cortisol levels in those who experienced EALs regardless of whether or not they had IBS. ${ }^{26}$ However, for patients with cortisol levels that returned to baseline more quickly the investigators noted decreased symptom severity $(r=-0.36, P<0.05)$ and better disease-specific QOL $(r=0.33, P<0.05)^{26}$ suggesting that perhaps the HPAA has a symptom-modulating effect.

Hormones released in response to stress, such as corticotropin-releasing hormone (CRH), regulate changes in gut motility, visceral perception and autonomic function. ${ }^{27} \mathrm{CRH}$ is released by the hypothalamus and stimulates adrenocorticotropic hormone (ACTH) release by the pituitary gland which in turn stimulates cortisol release from the adrenal cortex. ${ }^{28}$ Exogenous administration of $\mathrm{CRH}$ has been shown to increase colonic motility and stimulate serum ACTH with an exaggerated response found in patients with IBS. ${ }^{29}$ In a study by Fukudo, ${ }^{27} 10$ IBS patients and 10 control subjects underwent pressure transducer placement in the colon and were then injected with CRH. While there was no difference in motility at baseline, the IBS group developed significantly greater colonic motor activity and longer duration of abdominal symptoms in response to $\mathrm{CRH}$ administration compared with controls $(P<0.05)$. CRH induced a rise in serum ACTH in both groups, although a significantly higher increase in the IBS group $(P<0.01)$. There was no difference in serum cortisol response in the 2 groups. ${ }^{29}$

Ringel et $\mathrm{al}^{30}$ used PET scan to compare cerebral blood flow in patients with IBS and controls with post-hoc analyses comparing patients with a history of physical or sexual abuse and patients without abuse. ${ }^{30}$ They found a greater increase in anterior cingu- 
late cortex activity in non-IBS subjects and those without a history of abuse. IBS patients showed higher activity in the thalamus, an area previously shown to be associated with pain response.

The main strength of this study is the novel study population. There is a lack of previous investigation into differences among IBS patients of various races in the US. Our structured survey relied on standardized questionnaires including Rome III and therefore misclassification was minimized. ${ }^{31}$ Another strength is that our study group appeared to represent the target population after weighting and scaling. For example, our prevalence estimate for the proportion of adult AA in the ZTCA that are female was 56.8 (50.8-62.5) while the actual proportion is $58.8 \%$ (https:// www.census.gov/regions/philadelphia). The mean weighted age of our adult AA sample is 44.2 (42.0-46.4) years, similar to census results (ranging from 40.7 to 46.6 years). The study group had a low rate of college education. A recent survey found that only $9 \%$ of adults (not stratified by race) over the age of 25 had a college degree in the ZCTA; very close to the weighted prevalence found in our survey of 6.6 (4.1-10.4).

There are several limitations to our study which should be stressed. First, this study took place in an urban, low-income population with a relatively small sample size. The generalizability to other AA populations with different social and economic demographics must be considered with caution. Secondly, this study specifically addressed AA and cannot be extrapolated to other races or ethnicities. Additionally, data was obtained from self-reported questionnaires and lacks objective findings. Subjects were not required to have a colonoscopy, imaging, or other investigation to rule out organic causes for symptoms. A final potential weakness relates to our stratified sampling methodology and our failure to estimate the impact of the design effect on our prevalence estimates and sample size calculation. Estimating the design effect requires comparing the variance of the prevalence estimate under different sampling methods. While the variability of the prevalence estimate under simple random sampling can be derived from basic probability theory, multiple simulations to discover the variability under respondent-driven sampling such as our survey are required. ${ }^{32}$ Generally, but not always, the design effects from respondent-driven sampling are less precise than estimates from simple random sampling.

In conclusions, as with Caucasian subjects with IBS, the prevalence of previous emotional and/or physical trauma is substantial in the AA community. After adjustment for other important confounders, AA with IBS were twice as likely to suffer from PTSD. PTSD was associated with adverse lifestyle choices (drug and alcohol misuse) and had a substantial impact on QOL, both in the physical and particularly psychological domains. Our study highlights the need to screen for PTSD in AA with IBS.

\section{References}

1. Saito YA, Schoenfeld P, Locke GR 3rd. The epidemiology of irritable bowel syndrome in North America: a systematic review. Am J Gastroenterol 2002;97:1910-1915.

2. Hungin AP, Chang L, Locke GR, Dennis EH, Barghout V. Irritable bowel syndrome in the United States: prevalence, symptom patterns and impact. Aliment Pharmacol Ther 2005;21:1365-1375.

3. Grover M, Drossman DA. Centrally acting therapies for irritable bowel syndrome. Gastroenterol Clin North Am 2011;40:183-206.

4. Hungin AP, Whorwell PJ, Tack J, Mearin F. The prevalence, patterns and impact of irritable bowel syndrome: an international survey of 40,000 subjects. Aliment Pharmacol Ther 2003;17:643-650.

5. Andrews EB, Eaton SC, Hollis KA, et al. Prevalence and demographics of irritable bowel syndrome: results from a large web-based survey. Aliment Pharmacol Ther 2005;22:935-942.

6. Cain KC, Jarrett ME, Burr RL, Rosen S, Hertig VL, Heitkemper MM. Gender differences in gastrointestinal, psychological, and somatic symptoms in irritable bowel syndrome. Dig Dis Sci 2009;54: 1542-1549.

7. Wigington WC, Johnson WD, Minocha A. Epidemiology of irritable bowel syndrome among African Americans as compared with whites: a population-based study. Clin Gastroenterol Hepatol 2005; 3:647-653.

8. Kang JY. Systematic review: the influence of geography and ethnicity in irritable bowel syndrome. Aliment Pharmacol Ther 2005;21:663676.

9. El-Salhy M. Irritable bowel syndrome: diagnosis and pathogenesis. World J Gastroenterol 2012;18:5151-5163.

10. Minocha A, Bollineni D, Johnson WD, Wigington WC. Racial differences in general health, suicidal thoughts, physical and sexual abuse in African-Americans and Caucasians with irritable bowel syndrome. South Med J 2010;103:764-770.

11. Minocha A, Johnson WD, Abell TL, Wigington WC. Prevalence, sociodemography, and quality of life of older versus younger patients with irritable bowel syndrome: a population-based study. Dig Dis Sci 2006;51:446-453.

12. Tanaka Y, Kanazawa M, Fukudo S, Drossman DA. Biopsychosocial model of irritable bowel syndrome. J Neurogastroenterol Motil 2011; 17:131-139

13. Yuan YZ, Tao RJ, Xu B, et al. Functional brain imaging in irritable bowel syndrome with rectal balloon-distention by using fMRI. World J Gastroenterol 2003;9:1356-1360.

14. Drossman DA. Abuse, trauma, and GI illness: is there a link? Am J Gastroenterol 2011;106:14-25.

15. North CS, Hong BA, Alpers DH. Relationship of functional gastrointestinal disorders and psychiatric disorders: implications for treatment. World J Gastroenterol 2007;13:2020-2027.

16. Warner $\mathrm{CH}$, Warner CM, Appenzeller GN, Hoge CW. Identifying and managing posttraumatic stress disorder. Am Fam Physician 
2013;88:827-834.

17. Chou KL. Childhood sexual abuse and psychiatric disorders in middle-aged and older adults: evidence from the 2007 Adult Psychiatric Morbidity Survey. J Clin Psychiatry 2012;73:e1365-e1371.

18. Weiss NH, Tull MT, Lavender J, Gratz KL. Role of emotion dysregulation in the relationship between childhood abuse and probable PTSD in a sample of substance abusers. Child Abuse Negl 2013;37: 944-954.

19. Friedenberg FK, Makipour K, Palit A, Shah S, Vanar V, Richter JE. Population-based assessment of heartburn in urban Black Americans. Dis Esophagus 2013;26:561-569.

20. Ford AC, Bercik P, Morgan DG, Bolino C, Pintos-Sanchez MI, Moayyedi P. Validation of the Rome III criteria for the diagnosis of irritable bowel syndrome in secondary care. Gastroenterology 2013; 145:1262-1270, e1.

21. Ouimette P, Wade M, Prins A, Schohn M. Identifying PTSD in primary care: comparison of the Primary Care-PTSD screen (PCPTSD) and the General Health Questionnaire-12 (GHQ). J Anxiety Disord 2008;22:337-343.

22. Freedy JR, Steenkamp MM, Magruder KM, et al. Post-traumatic stress disorder screening test performance in civilian primary care. Fam Pract 2010;27:615-624.

23. Ware JE Jr, Gandek B. Overview of the SF-36 Health Survey and the International Quality of Life Assessment (IQOLA) Project. J Clin Epidemiol 1998;51:903-912.

24. Reding KW, Cain KC, Jarrett ME, Eugenio MD, Heitkemper MM. Relationship between patterns of alcohol consumption and gastrointestinal symptoms among patients with irritable bowel syndrome. Am J Gastroenterol 2013;108:270-276.
25. Halder SL, Locke GR 3rd, Schleck CD, Zinsmeister AR, Talley NJ. Influence of alcohol consumption on IBS and dyspepsia. Neurogastroenterol Motil 2006;18:1001-1008.

26. Videlock EJ, Adeyemo M, Licudine A, et al. Childhood trauma is associated with hypothalamic-pituitary-adrenal axis responsiveness in irritable bowel syndrome. Gastroenterology 2009;137:1954-1962.

27. Fukudo $\mathrm{S}$. Role of corticotropin-releasing hormone in irritable bowel syndrome and intestinal inflammation. J Gastroenterol 2007;42(suppl 17):48-51.

28. Bonfiglio JJ, Inda C, Refojo D, Holsboer F, Arzt E, Silberstein S. The corticotropin-releasing hormone network and the hypothalamicpituitary-adrenal axis: molecular and cellular mechanisms involved. Neuroendocrinology 2011;94:12-20.

29. Fukudo S, Nomura T, Hongo M. Impact of corticotropin-releasing hormone on gastrointestinal motility and adrenocorticotropic hormone in normal controls and patients with irritable bowel syndrome. Gut 1998;42:845-849.

30. Ringel Y, Drossman DA, Turkington TG, et al. Regional brain activation in response to rectal distension in patients with irritable bowel syndrome and the effect of a history of abuse. Dig Dis Sci 2003;48: 1774-1781.

31. Drossman DA, Dumitrascu DL. Rome III: New standard for functional gastrointestinal disorders. J Gastrointestin Liver Dis 2006;15: 237-241.

32. Wejnert C, Pham H, Krishna N, Le B, DiNenno E. Estimating design effect and calculating sample size for respondent-driven sampling studies of injection drug users in the United States. AIDS Behav 2012;16:797-806. 